\title{
Characteristics for A Flow Past A Circular Cylinder with Two Types of Radial Disturbances at $\operatorname{Re}=100$
}

\author{
Liming Lin ${ }^{\mathrm{a}}$, Xingfu Zhong ${ }^{\mathrm{b}}$, Yingxiang $\mathrm{Wu}^{\mathrm{c}}$ \\ Institute of Mechanics, Chinese Academy of Sciences, Beijing, China \\ allmbirthday@163.com, bxfzhong@imech.ac.cn, ‘yxwu@imech.ac.cn
}

Keywords: Radial disturbance; Circular cylinder; Vortex-induced vibration; Suppression; Riser.

\begin{abstract}
For better understanding physical mechanism of radial disturbances, previously proposed to disturb the wake flow and vortex-induced vibration of risers, numerical simulations are carried out for the flow around the fixed cylinder with such disturbances at a Reynolds number of 100 . After introducing disturbances, specific distributions of flow and vertical vorticity near cylinder surfaces are identified and related to different vortex patterns. The spanwise variation of free shear layers is discussed and physically explained. Different characteristics for the pressure on cylinder surfaces, frequency of vortex shedding and time history of lift are also analyzed and presented in detail.
\end{abstract}

\section{Introduction}

A large number of bluff bodies are commonly used in marine engineering, such as cables, risers, pipelines in subsea oil production. Due to the vortex shedding alternatively in the near wake, the bluff body undergoes fluctuating fluid forces. As the frequency of vortex shedding is close to the natural frequency of the body, the synchronization is occurred. The fluid force and oscillating amplitude of body would be suddenly amplified. Such phenomenon is called as the vortex-induced vibration (VIV). Its resulting fatigue damage has the potential to destroy the structural integrity, even the safety of people and production. In a half century, many documents have been published in investigating and understanding the dynamics of VIV. Comprehensive reviews can be referenced recently in $[1,2,3]$.

Thereby, many methods have been proposed over recent years for the aim of disturbing and controlling the flow around the body, leading to weaken, even totally suppress, the vortex shedding, as well as the fluctuating amplitude of the fluid force. For example, the surface control bumps was installed spirally around the body, proposed by Owen [4]. Almost $47 \%$ of drag reduction is realized. At certain wave steepness, defined by the ratio of the wave height to the wavelength, there is no signal of regular vortex shedding. Such device is effective mainly in the great mass-damping parameter. Up until now, streamline fairing [5] still exhibits very good aerodynamic performance due to its streamlined shape, resulting in the delay of the flow separation. Interactions between upper and lower free shear layers, as well as the formation of vortex shedding, can also been delayed effectively by splitter plates [6]. Because of variable ocean flow direction, the rotatable device is usually installed on streamline fairing and splitter plates. This could introduce a new trouble in dynamical instability. Helical strake $[7,8]$, the most widely used presently, is a device in disturbing spanwise uniformity of vortex shedding. Recently, triple-starting helical grooves were proposed as a new method in VIV suppression and drag reduction [9]. More information can be found out in reviews of $[10,11]$.

In addition, three-dimensional geometric disturbance, such a wavy front surface [12] and wholly wavy cylinders $[13,14]$, was introduced as another VIV suppressing method. Due to its sensitivity to the flow direction, a new idea that disturbance was introduced in a radial-spanwise plane, called radial disturbance, was proposed $[15,16]$ Preliminary experiments of oscillating pendulum with radial disturbance in water channel have shown that the oscillating amplitude at the start of synchronization is really reduced but increased subsequently at high velocity. Owing to obvious difference between pendulum and riser, the flow around the fixed cylinder with circular-section and wavy disturbances has been investigated at a Reynolds number of 100 [17]. The drag is increased with the increasing wave steepness. Lift in most cases is greatly reduced. Different flow patterns are classified. As for the 
moderate and strong effects of disturbance, some new vortex patterns are identified, including the complete suppression of Kármán vortex shedding. Nevertheless, there are still some unknown issues to be explored. It is helpful in better understanding the physical mechanism in effects of such disturbances on the wake flow.

As a basic study, the main purpose is to obtain the further knowledge about the effect of radial disturbance on the flow past a circular cylinder through the numerical simulation at $\boldsymbol{R} \boldsymbol{e}=100$. Harmonic and conic disturbances are considered with a series of wavelength and wave steepness. Firstly, the physical model is described briefly. Then effects of disturbances on the flow in the wake center plane, corresponding distribution of vertical vorticity on cylinder surfaces and in the near wake, the spanwise variation of free shear layers, time-averaged pressure coefficient on cylinder surface, Strouhal number and so on are presented and analyzed, respectively. At last, conclusions are given.

\section{Physcial Model}

Governing Equations. In an inertial Cartesian coordinate system $(x, y, z)$, an incompressible fluid flow past the circular cylinder with radial disturbances, as shown in Fig. 1, is considered. The peak and valley are defined as spanwise positions with the maximal and minimal diameters, respectively. Then, the wavy shape can be mathematically described by the wavelength $\lambda$ and the peak-to-valley wavy height $W$. The dimensionless forms of the continuity and Navier-Stokes equations are expressed as follows,

$$
\begin{gathered}
\nabla \cdot \boldsymbol{u}=0 \\
\frac{\partial \boldsymbol{u}}{\partial t}+(\boldsymbol{u} \cdot \nabla) \boldsymbol{u}=-\nabla p+\frac{1}{\boldsymbol{R} \boldsymbol{e}} \nabla^{2} \boldsymbol{u}
\end{gathered}
$$

where $\boldsymbol{u}$ is the velocity vector $(u, v, w), p$ is the static pressure, $t$ is non-dimensional time, $\boldsymbol{R} \boldsymbol{e}$ is the Reynolds number defined as $U_{\infty} D / v$ based on the free-stream velocity $U_{\infty}$, the diameter of cylinder $D$ and the kinematic viscosity of fluid $v$, and $\nabla$ is the gradient operator. In the periodic flow, the frequency of vortex shedding, $f$, is non-dimensionalized by the Strouhal number, that is $S t=f D / U_{\infty}$.

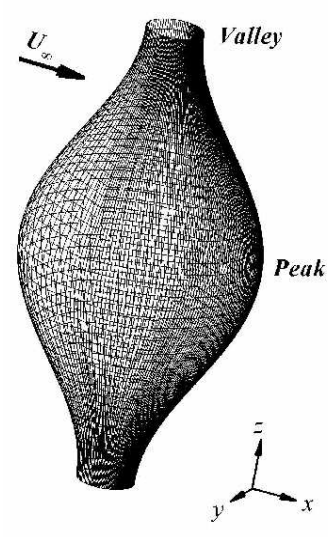

(a)

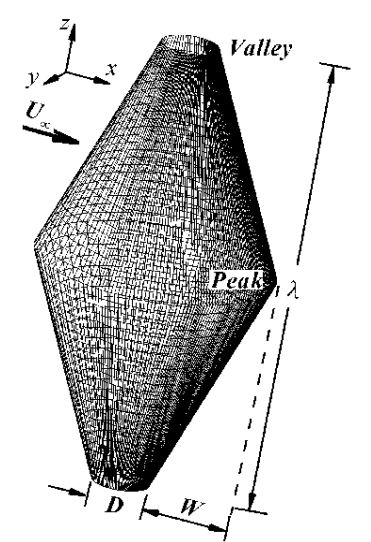

(b)

Figure 1 Schematics of a flow past circular cylinders with (a) harmonic and (b) conic disturbances.

Numerical Method. Computations are run by the Fluent software. With the assumption of the periodic flow along the span, boundary conditions in $(x, y)$ plane are described as following: the uniform free-stream velocity at inlet, the non-reflecting outflow at outlet, the free slip at vertical sides and non-slip on cylinder surfaces. The non-dimensional computational domain in $(x, y)$ plane is $40 \times 20$. The magnitude of convergent error for the continuity equation is less than $10^{-3}$.

As a basic case for comparison, two-dimensional flow past the circular cylinder is carried out firstly. The wake is typically described by the Kármán vortex shedding. The Strouhal number, $\boldsymbol{S t}$, is 
0.165 . The mean drag coefficient is 1.39 , and the RMS lift coefficient 0.256 . Through compared with previous experiments [18] and numerical results [19], the present calculations are acceptable for further study

\section{Features of Disturbance Effects}

In the flow past the straight cylinder, the main control parameter is the Reynolds number. As for the wavy shape, two independent length parameters are defined as the non-dimensional wavelength, $\lambda / D$, and wave steepness, $W / \lambda$. In the present study, a series of computational investigations have been carried out for different values of $\lambda / D$ with 4,6 and 8 and $W / \lambda$ with $0.0125,0.025,0.05,0.1$ and 0.2 .

Flow in The Wake Center Plane. As the flow approaches the cylinder, due to the peak on the most upstream position, the flow would be three-dimensional and directs from the peak to valley, as shown in Fig. 2. After the flow surrounds both sides of cylinder, there are mainly two types of flow patterns identified in the wake center plane. For most of cases with the vortex shedding alternatively, the spanwise flow behind the rear surface is also directed from the peak to valley, as shown in Fig. 2(a). And in the near wake, the flow is simply pointed to the downstream. Meanwhile, the spanwise velocity at certain spanwise positions, such as $z=\lambda / 4$ and $3 \lambda / 4$, is almost with the same sign along the downstream. However, as shown in Fig. 2(b) for the strong effect of disturbance, except the $\Omega$-type vortex shed at the valley, the flow direction behind the rear surface is from the valley to peak. The sign of spanwise velocity along the downstream is converse near the rear surface. Therefore, the recirculation region in the wake center plane is formed.

Correspondingly, different distribution of vertical vorticity on front and rear surfaces and in the near wake is also able to be described similarly for above two cases, as shown in Fig. 3. For example, in the first flow case, the sign of vertical vorticity on the rear surface is opposite to that on the front surface, and almost same to that in the near wake. But as for the second case, $\omega_{y}$ is always kept the same sign around the surface at the certain spanwise position, and changed its direction downstream.

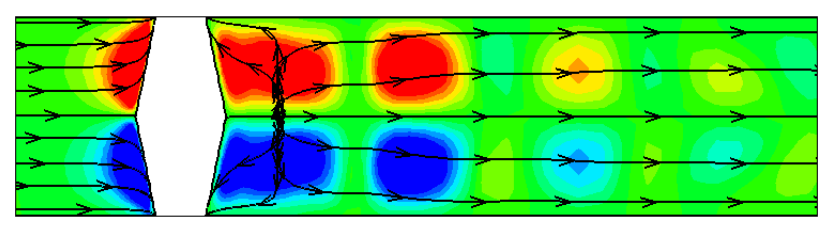

(a)

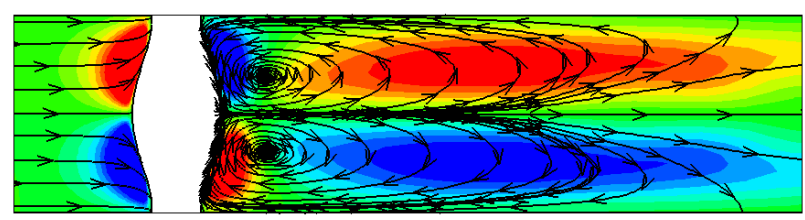

(b)

Figure 2 Streamlines and contours of spanwise velocity in the wake center plane, $(x, z)$, for (a) conic and (b) harmonic cylinders with $\lambda / D=4$ and $W / \lambda=0.1$, where the red and blue colors denote the positive and negative signs, respectively.

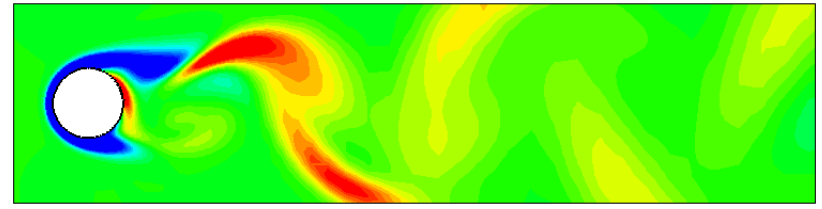

(a)

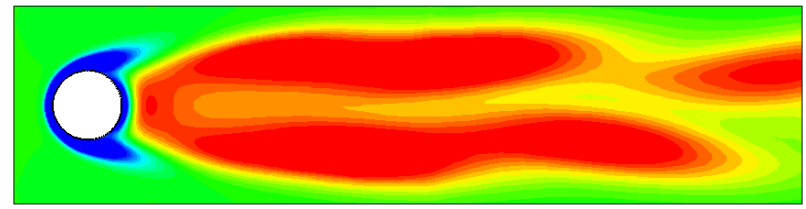

(b)

Figure 3 Contours of vertical vorticity at $z=\lambda / 4$ for (a) conic and (b) harmonic cylinders with $\lambda / D=4$ and $W / \lambda=0.1$, where the red and blue colors denote the positive and negative signs, respectively.

Free Shear Layers in The Near Wake. In previous work [17], the free shear layers, described by the contours of spanwise vorticity, are almost invariant along the span for the weak effect of disturbance. That is, the wake width is almost constant along the spanwise direction, as shown in Fig. 4(a). When the radial disturbance is moderate, the upper and lower shear layers at the peak and close to body becomes apart from each other, mainly attributed to larger diameter at the peak, typically as shown in Fig. 4(b). But, the end of shear layers, rolling up to form the shedding vortex, is away from each other at the valley. Such phenomenon is also appeared in cases with strong disturbance, typically as shown in Fig. 4(c) for the shear layers stretching further downstream, except those with the $\Omega$-type vortex 
shed at the valley with a little complex variation, as shown in Fig. 4(d). Such varied wake width can be explained and analyzed by the distribution of vertical vortex pairs in the near wake, schematically as shown in Fig. 5. The additional streamwise velocities at the peak and valley are induced with opposite directions by the vertical vortex pairs with specific distribution in the near wake. As a result, the streamwise velocity would be increased at the peak and reduced at the valley. The wake profile, described by the streamwise velocity varied along the vertical direction at the certain downstream position, would be enlarged at the valley, but shrunk at the peak. Then the wake width is reduced at the peak and increased at the valley.

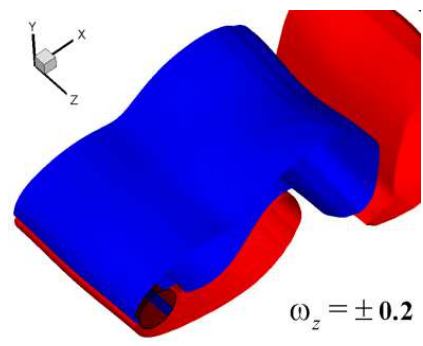

(a)

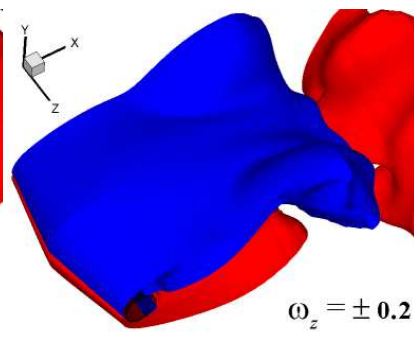

(b)

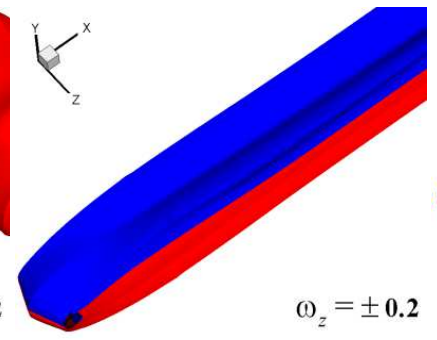

(c)

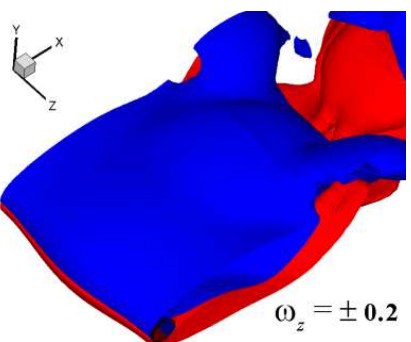

(d)

Figure 4 Iso-surfaces of spanwise vorticity of (a) harmonic cylinder with $\lambda / D=4$ and $W / \lambda=0.0125$, (b) conic cylinder with $\lambda / D=6$ and $W / \lambda=0.1$, (c) conic cylinder with $\lambda / D=4$ and $W / \lambda=0.2$, and (d) harmonic cylinder with $\lambda / D=8$ and $W / \lambda=0.05$, where the red and blue denote the positive and negative signs.

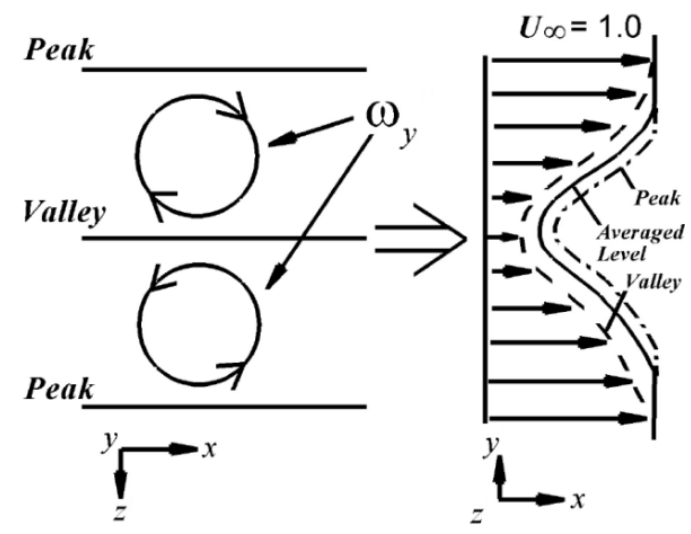

Figure 5 Schematics of relationship between specific distributions of vertical vortex pairs and wake profile or width varied along the span.

Pressure Coefficient on Cylinder Surfaces. The original idea of radial disturbance proposed in disturbing fluid force stems from the Bernoulli's equation. As stated above, the spanwise flow near the front of cylinder would be greater close to $z=\lambda / 4$ and $3 \lambda / 4$. Then the pressure would be reduced obviously, typically as shown in Fig. 6(a). However, due to the complex vortex shedding in the near wake, the distribution of pressure on rear surfaces would be apparently different from that on front surfaces. As shown in Fig. 6(b), (c) and (d), three situations are identified, based on the absolute value of pressure coefficients less than, equivalent to and greater than that of the straight cylinder. The relationship between the disturbance intensity and the distribution of pressure on rear surfaces is then summarized in Table 1. It can be seen that the pressure on rear surfaces for the weak disturbance with $\lambda / D=6$ and 8 , the moderate effect in Regime II-A and the strong influence in Regime III-A with the $\Omega$-type vortex shed on the valley and Regime III-C is really reduced, but increased in cases of the weak disturbance with $\lambda / D=4$ and the moderate effect in Regime II-B.

Howbeit, although the pressure on cylinder surfaces in some cases is indeed reduced, the drag of all disturbed cylinders is greater than that of the straight cylinder, as reported in previous study [17]. The reason of such discrepancy is the projected area of disturbed cylinders. As shown in Fig. 7, with the increase of wave steepness and wavelength, the increase of projected area, equal to $W \lambda$, is linearly rising up and quickly overcomes the decrease of pressure, whatever for the harmonic or conic cylinders. 


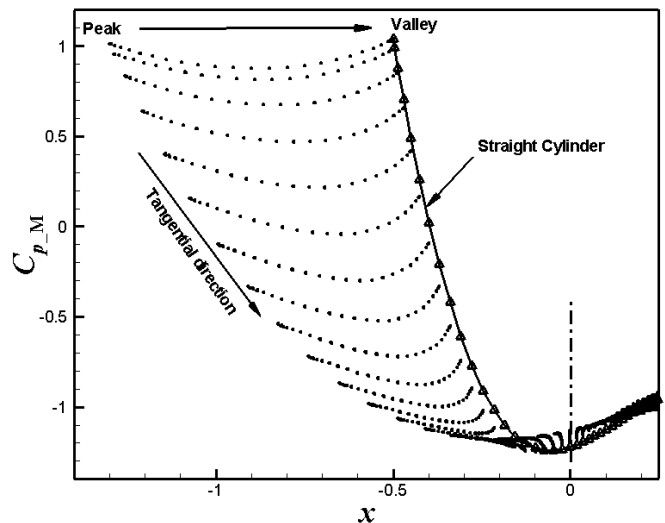

(a)

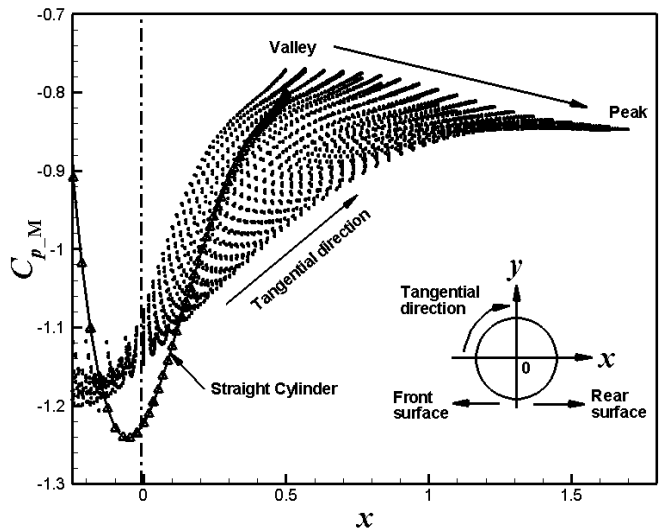

(c)

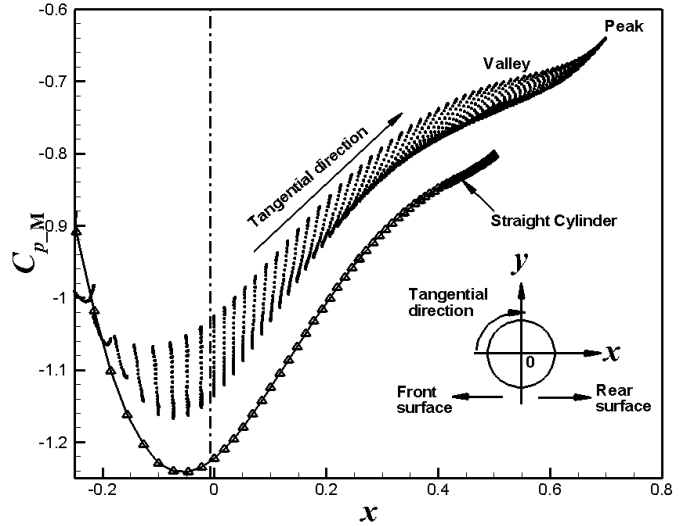

(b)

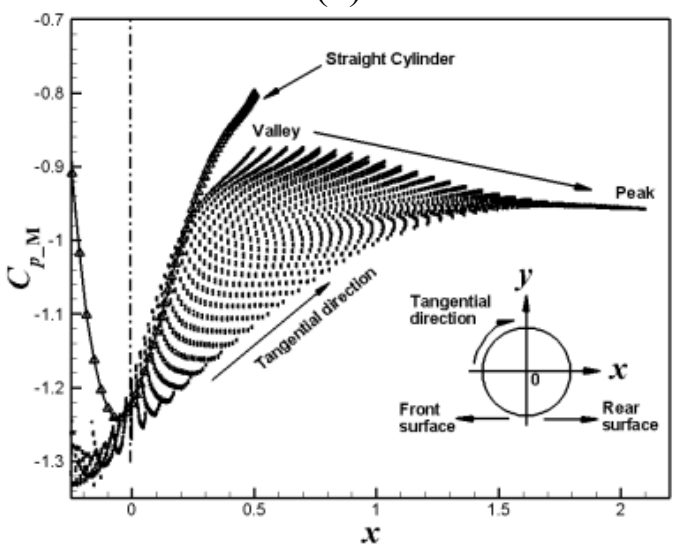

(d)

Figure 6 Variation of the time-averaged pressure coefficients, $C_{p \_\mathrm{M}}$, on (a) the front surface of harmonic cylinder with $\lambda / D=8$ and $W / \lambda=0.1$, rear surfaces of $(\mathrm{b})$ harmonic cylinder with $\lambda / D=8$ and

$W / \lambda=0.025$, (c) conic cylinder with $\lambda / D=6$ and $W / \lambda=0.2$, and (d) conic cylinder with $\lambda / D=8$ and $W / \lambda=0.2$.

Table 1. Relationship between the disturbance and pressure on rear surfaces for disturbed cylinders, where $\mathrm{L}, \mathrm{E}$ and $\mathrm{G}$ denote the less, equivalent and greater values of pressure, respectively, compared to that on the straight cylinder surface.

\begin{tabular}{|c|c|c|c|c|c|c|c|c|}
\hline $\begin{array}{c}\text { Disturbance } \\
\text { intensity }\end{array}$ & \multicolumn{2}{|c|}{ Weak } & \multicolumn{2}{c|}{ Moderate } & \multicolumn{3}{c|}{ Strong } \\
\hline $\begin{array}{c}\text { Regime } \\
\text { (condition) }\end{array}$ & $\begin{array}{c}\mathrm{I} \\
(\lambda / D=4)\end{array}$ & $\begin{array}{c}\mathrm{I} \\
(\lambda / D=6,8)\end{array}$ & $\mathrm{II}-\mathrm{A}$ & $\mathrm{II}-\mathrm{B}$ & $\begin{array}{c}\text { III-A } \\
(\Omega \text { vortex on } \\
\text { peak })\end{array}$ & $\begin{array}{c}\text { III-A } \\
(\Omega \text { vortex } \\
\text { on valley })\end{array}$ & III-B & III-C \\
\hline $\begin{array}{c}\text { Comparison of } \\
\text { surface pressure }\end{array}$ & $\mathrm{G}$ & $\mathrm{L}$ & $\mathrm{L}$ & $\mathrm{G}$ & $\mathrm{E}$ or G & $\mathrm{L}$ & $\mathrm{E}$ & $\mathrm{L}$ \\
\hline
\end{tabular}

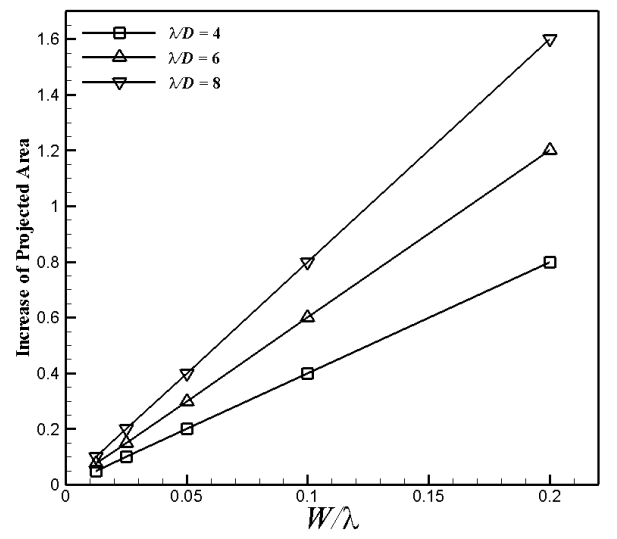

Figure 7 Increase of projected area of disturbed cylinders versus the wave steepness and wavelength. 
Strouhal Number. After introducing radial disturbances, the original vortex pattern, Kármán vortex shedding, is gradually disturbed and even totally disappeared. Several different vortex formations are identified, such as the $\Omega$-type vortex and cloud-like vortex. Hence, the frequency of such vortex shedding would be changed. As shown in Fig. 8, the Strouhal number, the main frequency of the time history of lift coefficient, is generally decreased as the wave steepness increases, whatever for the harmonic or conic cylinders. Such feature could be helpful in design of some device only required to suppress the oscillating frequency.

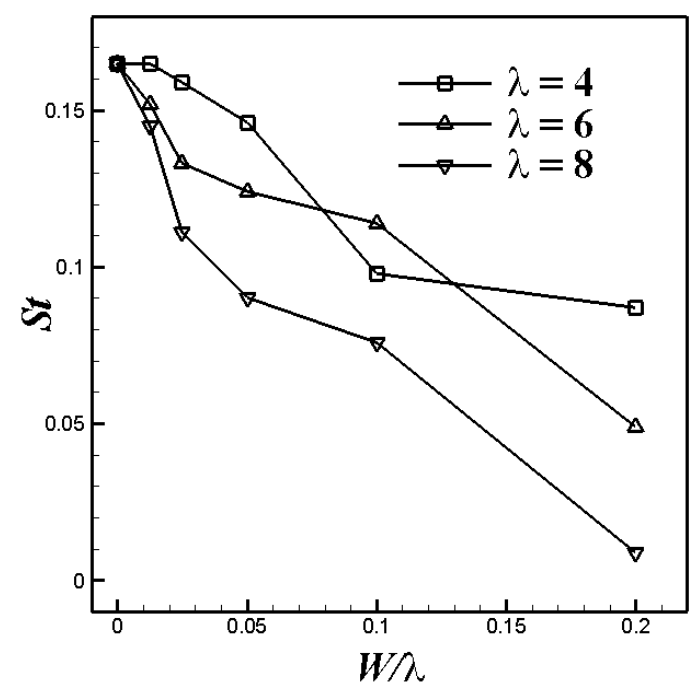

(a)

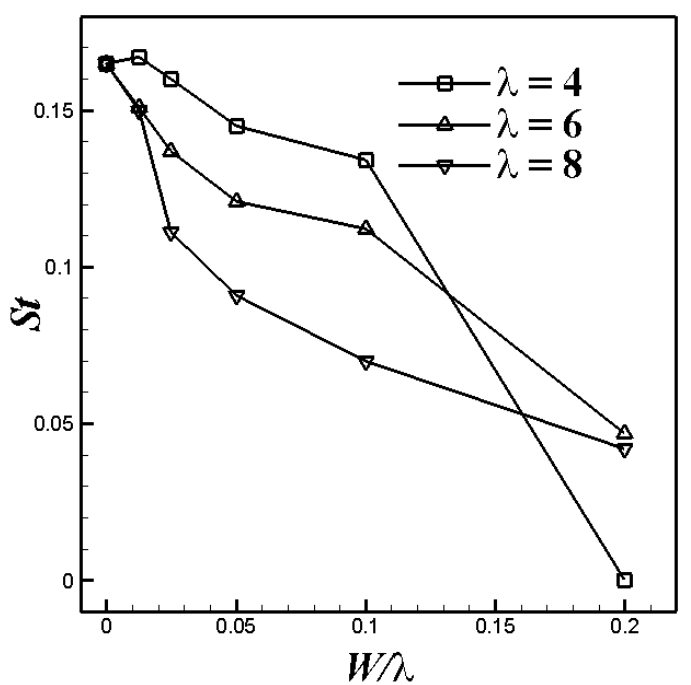

(b)

Figure 8 Variation of the Strouhal number with the wave steepness and wavelength for (a) harmonic and (b) conic cylinders.

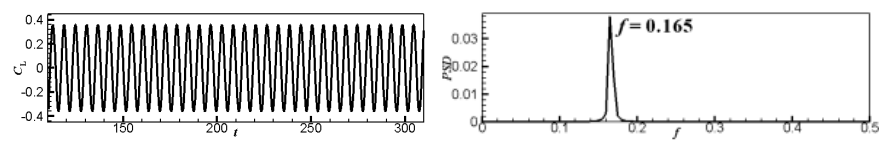

Figure 9 The time history of lift coefficient and frequency analysis for the straight cylinder.

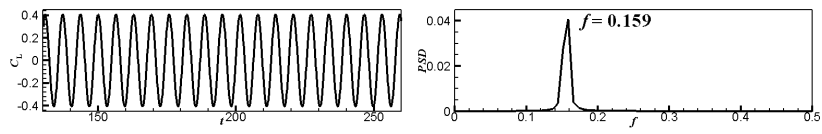

(a)

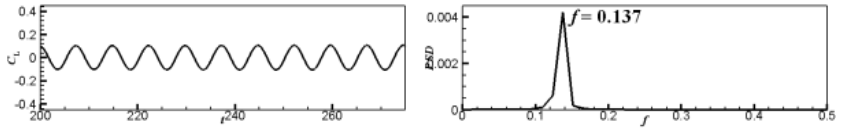

(b)

Figure 10 Typical frequency analysis of lift coefficient for (a) the harmonic cylinder with $\lambda / D=4$ and $W / \lambda=0.025$ in the weak disturbance of Regime I and (b) the conic cylinder with $\lambda / D=6$ and $W / \lambda=0.025$ in the moderate disturbance of Regime II-A.

Time History of Lift Coefficient. At $\boldsymbol{R} e=100$, the wake flow past the straight cylinder is two-dimensional and typically described by the Kármán vortex with a single frequency of shedding, as shown in Fig. 9. With the introduction of radial disturbances, the lift still remains the steady fluctuation with a single frequency for the weak disturbance with $\lambda / D=4$ and 6 , and the moderate disturbance in Regime II-A, as shown in Fig. 10. However, with further increasing disturbance intensity, the lift is no longer kept with constant amplitude. As shown in Fig. 11, the peak of oscillating lift is wavily modified along the time and with several frequencies.

Through the wavelet analysis, some different features of oscillation frequency varied with time are obtained. As shown in Fig. 11(a) for the weak disturbance with $\lambda / D=8$, the modification of fluctuating amplitude of lift is accompanied with mixed several frequencies. When the peak of lift reaches the minimum, the frequency is also reduced. But, as for the moderate effect in Regime II-B, as shown in Fig. 11(b), only main frequency is appeared during the process of amplitude modification, while there are two frequencies occurred at the minimum of lift amplitude. When the disturbance becomes strong, the oscillating lift sometimes is similar to that with a single frequency, such as cases for 
harmonic cylinder at $\lambda / D=4$ and $W / \lambda=0.1$ with very weak oscillation in Regime III-C and $\lambda / D=8$ and $W / \lambda=0.05$ in Regime III-A with the $\Omega$-type vortex shed at the valley. In other certain cases, as shown in Figs. 11(c) and (d), the variation of frequency is obviously different from above, such as certain oscillation frequency only appeared in the certain period. On the other hand, it could be predicted that the lower frequency of amplitude modification with a longer period would be identified if the computational time is longer enough.

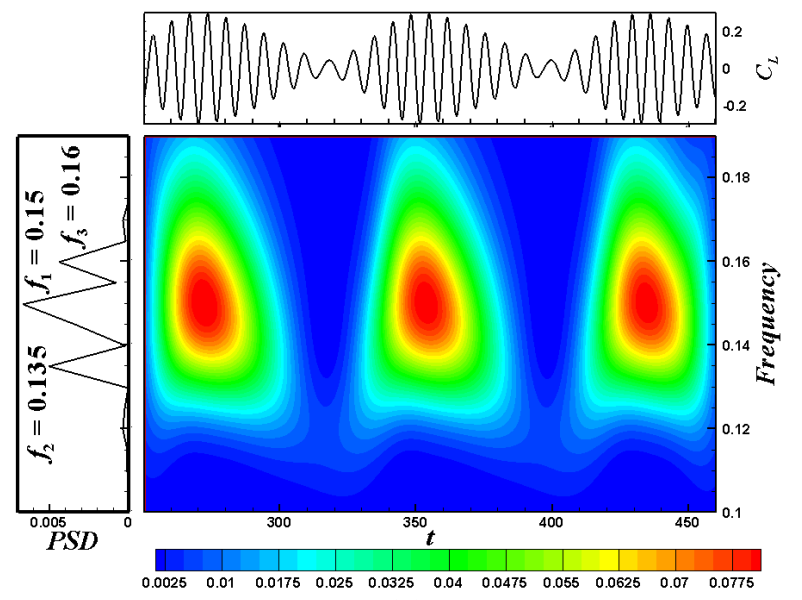

(a)

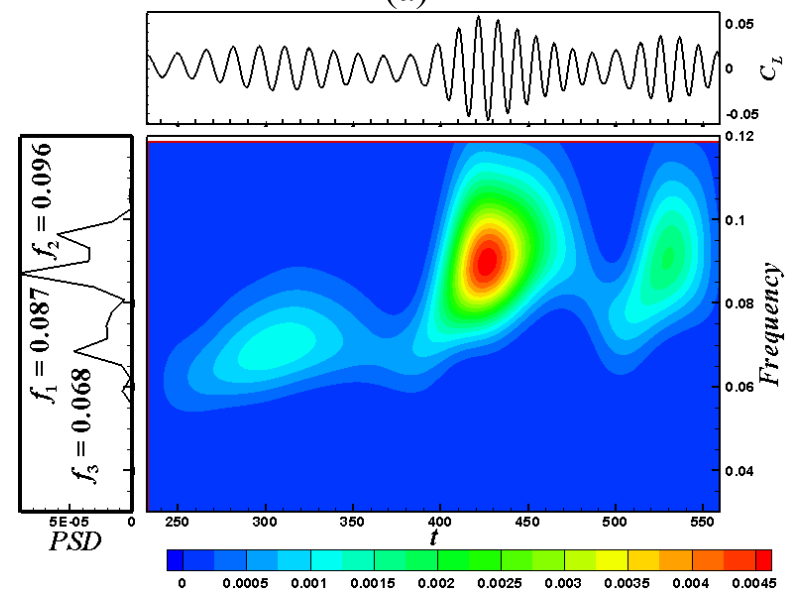

(c)

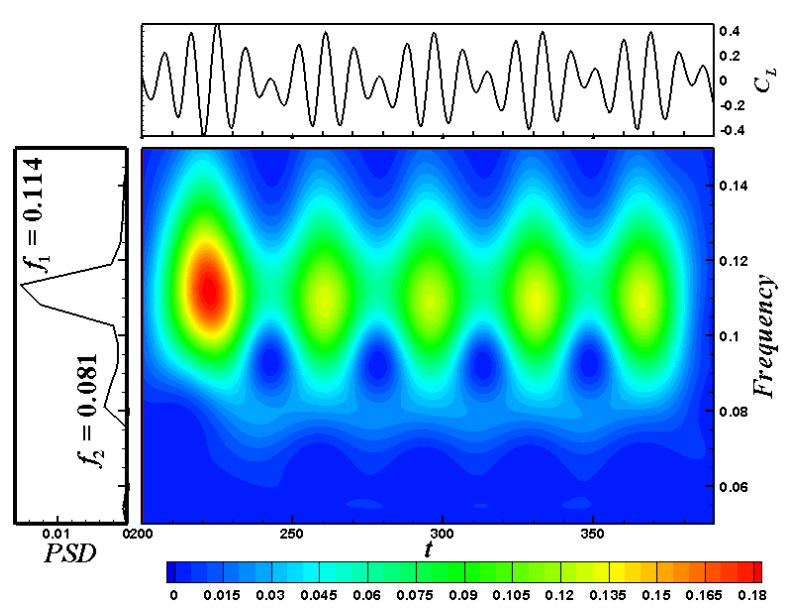

(b)

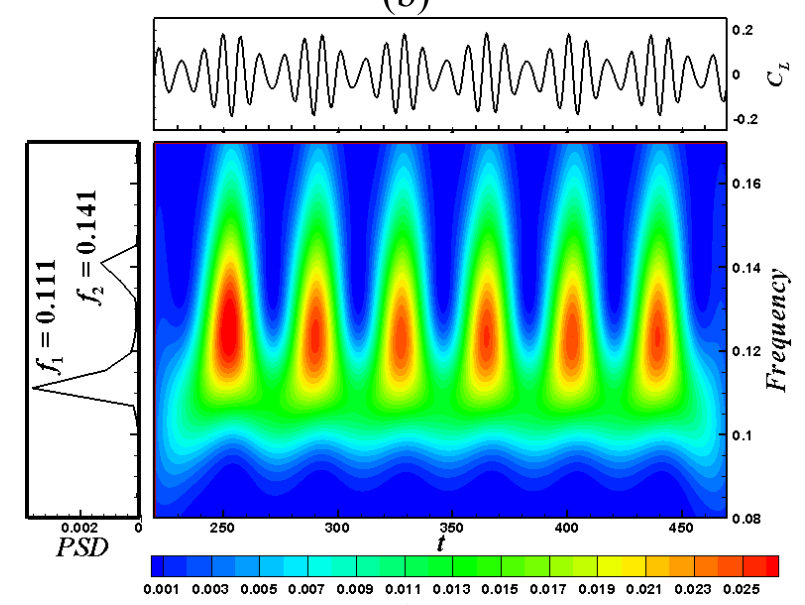

(d)

Figure 11 The wavelet analysis for time history of lift coefficient for (a) conic cylinder with $\lambda / D=8$ and $W / \lambda=0.0125$ in the weak effect of Regime $\mathrm{I}$, (b) harmonic cylinder with $\lambda / D=6$ and $W / \lambda=0.1$ in the moderate disturbance of Regime II-B, (c) harmonic cylinder with $\lambda / D=4$ and $W / \lambda=0.2$, and (d) conic cylinder with $\lambda / D=8$ and $W / \lambda=0.025$.

\section{Summary}

In present paper, numerical simulations are carried out to explore more characteristics of flows past the circular-section cylinder with harmonic and conic disturbances at the Reynolds number of 100.

As the flow approaches the cylinder, the spanwise component of velocity always directs from the peak to valley on the front cylinder. While on the rear cylinder, the flow direction is just converse, that is from the valley to peak, for the strong disturbance except cases with the $\Omega$-type vortex shed at the valley. Due to the specific distribution of spanwise velocity, as well as the vertical vorticity, the recirculation could be formed in the wake center plane. Furthermore, the existence of vertical vortex pairs in the near wake leads to the wavy variation of free shear layers along the span.

Similarly, different distributions of pressure coefficient on cylinder surfaces are distinguished, related with the corresponding vortex patterns in the near wake. And the relationship between the distribution of pressure and the disturbance intensity is qualitatively summarized. 
Generally, the frequency of vortex shedding is gradually decreased with the increasing wave steepness. And there is the amplitude modification in time history of lift coefficient. In some cases, such as weak disturbance with $\lambda / D=4$ and 6 , there is only one frequency with steady fluctuating amplitude. But in some cases, such as the strong disturbance, multi-frequencies with the wavy fluctuating amplitude of lift are also appeared. As for the later, in the period of amplitude modification, the lift is fluctuated with the main frequency or mixed with multi-frequencies through the wavelet analysis.

In future, due to the natural difference between the near wake flows at lower and higher Reynolds numbers, it is necessary to investigate the effects of such radial disturbances on hydrodynamic parameters and the near wake flow at higher Reynolds numbers.

\section{Acknowledgements}

The authors sincerely acknowledge the support of the National Key Scientific Instrument and Equipment Development Program of China (No. 2011YQ120048) for the work reported in this paper.

\section{References}

[1] T. Sarpkaya: J. Fluid Struct. Vol. 10 (2004), p. 389

[2] C.H.K. Williamson and R. Govardhan:. Annu. Rev. Fluid Mech. Vol. 36 (2004), p. 413

[3] R.D. Gabbai and H. Benaroya: J. Sound Vib. Vol. 282 (2005), p. 575

[4] J. C. Owen, P.W. Bearman and A.A. Szewczyk: J. Fluid Struct. Vol. 15 (2001), p. 597

[5] L. Lee and D.W. Allen: Offshore Tech Conf Houston, Texas, USA, OTC 17125 (2005)

[6] G.R.S. Assi, P.W. Bearman and N. Kitney: J. Fluid Struct. Vol. 25 (2009), p. 666

[7] A.D. Trim, H. Braaten, H. Lie and M.A. Tognarelli: J. Fluid Struct. Vol. 21 (2005), p. 335

[8] I. Korkischko and J.R. Meneghini: J. Fluid Struct. Vol. 26 (2010), p. 611

[9] S. Huang: J. Fluid Struct. Vol. 27 (2011), p.1124

[10]T. Sarpkaya and M. Isaacson: Mechanics of wave forces on offshore structures, published by Van Nostrand Reinhold Company, New York (1981), p. 606

[11]R.A. Kumar, C.H. Sohn and B.H.L. Gowda: Recent Patents on Mechanical Eng, Vol. 1 (2008), p. 1

[12]P.W. Bearman and J.C. Owen: J. Fluid Struct. Vol. 12 (1998), p. 123

[13] J.C. Owen, A.A. Szewczyk and P.W. Bearman: Bulletin of the American Physical Society Vol. 44 (1999), p. 124

[14]L.M. Lin, G.C. Ling and Y.X. Wu: Chin. Phys. Lett. Vol. 27 (2010), p. 034702

[15] L.M. Lin, X.F. Zhong and Y.X. Wu: Proc 21st Int Offshore and Polar Eng Conf, ISOPE, Maui (2011), p. 1283

[16] L.M. Lin, X.F. Zhong and Y.X. Wu: Proc 22nd Int Offshore and Polar Eng Conf, ISOPE, Rhodes (2012), p. 623

[17] L.M. Lin, X.F. Zhong and Y.X. Wu: Proc 23rd Int Offshore and Polar Eng Conf, ISOPE, Anchorage (2013), p. 387

[18] C.H.K. Williamson: Annu. Rev. Fluid. Mech. Vol. 28 (1996), p. 477

[19] D.J. Newman and G.E. Karniadakis: J. Fluid Mech. Vol. 344 (1997), p. 95 
Applied Mechanics, Fluid and Solid Mechanics

10.4028/www.scientific.net/AMR.871

Characteristics for a Flow Past a Circular Cylinder with Two Types of Radial Disturbances at $\boldsymbol{R} \boldsymbol{e}=\mathbf{1 0 0}$ 10.4028/www.scientific.net/AMR.871.107 